\title{
Long non-coding RNA HOXA11-AS promotes cell proliferation and metastasis in human breast cancer
}

\author{
JIAN-CHUN SU and XUE-FENG HU
}

\begin{abstract}
Department of Head and Neck Radiotherapy, The First People's Hospital of Foshan, Foshan, Guangdong 528000, P.R. China
\end{abstract}
Received November 19, 2016; Accepted May 31, 2017

DOI: $10.3892 / \mathrm{mmr} .2017 .7163$

\begin{abstract}
Breast cancer is one of the most frequently occurring malignancies in female cancers worldwide, however, its detailed mechanism of tumorigenesis remains to be elucidated. Long non-coding RNAs (LncRNAs) have previously been demonstrated to be important in multiple cancers, including breast cancer. The present study aimed to elucidate the molecular mechanism of the effects of the novel Lnc RNA HOXA11-AS, on cell proliferation and metastasis in breast cancer. The data revealed that the relative transcript level of HOXA11-AS was upregulated in vivo and in vitro in models of breast cancer. Knockdown of HOXA11-AS in MDA-MB-231 and MDA-MB-436 breast cancer cell lines inhibited the formation of cell colonies and arrested the cell cycle at the G0/G1 phase. Depletion of HOXA11-AS using two specific short interfering (si)RNAs against HOXA11-AS (siHOXA11-AS-1 and siHOXA11-AS-2) additionally suppressed the cell proliferative rate. Furthermore, transwell assays and wound-healing analysis revealed that siRNA transfection inhibited cell migration and invasion by $\sim 50 \%$ in the two cell lines. The results of the present study demonstrated the oncogenic role of HOXA11-AS in breast cancer, providing novel clues for the future clinical diagnosis and treatment of early stage breast cancer patients.
\end{abstract}

\section{Introduction}

Breast cancer is among the most common reasons of cancer-related deaths for females worldwide and its incidence increases with age $(1,2)$. The average age of breast cancer patients is 61 years when they are diagnosed first time, and the majority of deaths occurs after the age of 65 years $(3,4)$. Higher noncompliance for clinical treatment, presence of

Correspondence to: Dr Xue-Feng Hu, Department of Head and Neck Radiotherapy, The First People's Hospital of Foshan, Building No. 2, 81 North Lingnan Road, Shancheng, Foshan, Guangdong 528000, P.R. China

E-mail: xuefeng196hu@163.com

Key words: long non-coding RNA, homeobox A11-AS, breast cancer, proliferation, metastasis comorbidities contributing to mortality as well as the higher cost of the treatment are three main factors that explain why patients over 65 years are not suitable for screening strategies for breast cancer $(5,6)$. Therefore, it is urgent to find the sensitive gene for potential breast cancer patients and diagnosis the patients in an early stage.

Long non-coding RNAs (LncRNAs) are RNAs that have a full length of more than $200 \mathrm{bp}$ nucleotides and do not encode for proteins (7). A variety of LncRNAs are found to play significant roles in the process of tumorigenesis of multiple cancers, including breast cancer $(8,9)$. For instance, LncRNA Xist was downregulated in breast cancer and increased AKT phosphorylation via HDAC3-mediated repression of PHLPP1 expression (10); LncRNA LINP1 regulated repair of DNA double-strand breaks in triple-negative breast cancer (11).

The homeobox A11 (HOXA11) gene is a member of HOX gene clusters which feature prevalent intergenic transcription between coding genes and stimulate embryonic development in human beings and mice $(12,13)$. LncRNA HOXA11-AS transcripts occur in the adult human endometrium and varies throughout the menstrual cycle (14). When the transcript level of HOXA11-AS is suppressed, the expression of HOXA11 increases, indicating its role in regulating mRNA expression. HOXA11-AS inhibits the expression of HOXA11 by competing for the transcription of a common gene $(15,16)$. Functionally, HOXA11-AS showed its potent effects on cell proliferation in gastric cancer (17) and glioma (18), indicating its critical role in human tumorigenesis.

In our study, we aimed to investigate the role of HOXA11-AS in human breast cancer. To this end, human clinical tumor tissues were collected and included for subsequent RT-PCR analysis. Cell cycle analysis and colony formation, cell proliferation assays were performed to reveal the role of HOXA11-AS in cell proliferation in breast cancer. Transwell assays and wound-healing analysis were included to explore the effects of HOXA11-AS on cell metastasis. Our findings are the first to uncover the importance of HOXA11-AS in breast cancer, indicating that HOXA11-AS might work as a novel tumor biomarker in the early stage of breast cancer diagnosis and treatment.

\section{Materials and methods}

Human tissues. This study was approved by the Ethics Committee of the First People's Hospital of Foshan (Foshan, 
China) and all procedures were conducted following the instructions. A total of 100 breast cancer patients were included and their tumor tissues as well as the adjacent non-cancerous tissues were dissected and immediately frozen into the liquid nitrogen. The breast cancer patients included were diagnosed by at least two independent pathologists by paraffin slices. No chemotherapies or radiotherapies were accepted before the surgery. Clinical characteristics such as age, marriage status and TNM staging, and the pathological variables, including Ki67, ER, PR, and HER2 were collected. Any patient with missed clinicopathological information was excluded from this study. All patients showed their full intention to participate into our study and written consent was obtained from each patients.

Cell culture and transfection. Normal breast epithelial cell line MCF10A was purchased from American Type Culture Collection (ATCC; Manassas, VA, USA, USA) and incubated as per the manufactures' protocols. Four breast cancer cell lines MDA-MB-231, MDA-MB-436, MCF7 and T47D were commercially from the Cell Bank of Chinese Academy of Sciences (Shanghai, China). All of the cells were cultured in the dulbecco's modified eagle medium (DMEM; Gibco, Grand Island, NY, USA) supplied with $10 \%$ fetal bovine serum (FBS; Gibco) at $37^{\circ} \mathrm{C}$ supplied with $5 \% \mathrm{CO}_{2}$. The Culture medium were refreshed once two days unless otherwise stated. Two specific siRNA against HOXA11-AS (termed as siHOXA11-AS-1 and siHOXA11-AS-2) were synthesized and a negative control siRNA (siNC) was also included for control. Cell transfection was applied by using Lipofectamine ${ }^{\circledR} 2000$ (Invitrogen Life Technologies, Carlsbad, CA, USA) when the cell confluence reached approximately $70 \%$.

RNA extraction and polymerase chain reaction. Total RNAs from both clinical tissues and cultured cells were extracted by Trizol reagent (Takara Bio, Dalian, China) with a volume of $1 \mathrm{ml}$ for each well in 6-well plates. RNAs were quantified and qualified by Nanodrop (Thermo Fisher Scientific, Inc., Waltham, MA, USA). A total of $1 \mu \mathrm{g}$ RNA was reversely transcribed into cDNA with AMV reverse transcriptase according to the standard protocols (Roche Applied Science, Mannheim, Germany). Afterwards, polymerase chain reaction (PCR) was performed with an ABI 7900 machine using a PCR-mix with hot start Taq DNA polymerase and SYBR-Green (Roche Applied Science). Delta CTs were normalized to GAPDH control gene and delta delta CT analysis were performed to calculate relative expression of RNA from each sample.

Colony formation assay. Both MDA-MB-231 and MDA-MB-436 cells were seeded in 6-well plates and pre-treated with specific siRNAs against HOXA11-AS and $48 \mathrm{~h}$ later, were spread into 12 -well plates (100 cells/well) in triplicate. Afterwards, the plates were incubated at $37^{\circ} \mathrm{C}$ for continuous 14 days and the colonies were fixed with pre-iced methanol and stained with crystal violet (1\%) for $5 \mathrm{~min}$. Colonies that contains more than 50 cells were counted as survivors under a Nikon microscope (Nikon Corporation, Tokyo, Japan; magnification, x200).
Cell cycle analysis. Both MDA-MB-231 and MDA-MB-436 cells were seeded into 6-well plates (approximately $3 \times 10^{5}$ cells/well) and pre-treated with siRNAs against HOXA11-AS for $48 \mathrm{~h}$ when a confluence of $85 \%$ was reached. Then, cells were collected by low-speed centrifugation $(1,000 \mathrm{rpm})$ for $5 \mathrm{~min}$ and cell pellets were resuspended in $1 \mathrm{ml}$ of PBS, fixed in $75 \%$ of ice-cold ethanol and incubated at $-20^{\circ} \mathrm{C}$ for another $48 \mathrm{~h}$. Prior to flow cytometry (FCM) analysis, MDA-MB-231 and MDA-MB-436 cells were lysed, centrifuged and re-suspended in propidium iodide (PI) staining buffer supplied with $50 \mu \mathrm{l} / \mathrm{ml}$ of PI and $250 \mu \mathrm{l} / \mathrm{ml}$ of RNase A. Afterwards, cell mixtures were incubated at $4^{\circ} \mathrm{C}$ for additional $30 \mathrm{~min}$ in the dark environment and finally examined by fluorescence activated cell sorting (FACS) technique (Beckman Coulter, Krefeld, Germany).

Cell proliferation assay. MDA-MB-231 and MDA-MB-436 cells were seeded into chamber slides and transfected with specific siRNAs against HOXA11-AS and cultured in $37^{\circ} \mathrm{C}$ incubator under the selection of G418. EdU nucleoside analogue was allowed to incorporate during DNA synthesis for an hour and cells were processed with Click-iT EdU Imaging kit (Thermo Fisher Scientific, Inc.) following the protocols. Relative proliferative rate was assessed by taking mean and standard deviations of five non-biased image fields. To evaluate the overall survival, a total of $3 \times 10^{4}$ cells were seeded into each well in 12-well plates and transfected with siRNAs in triplicate. After selection of 6 days, overall survival was explored with $0.5 \mathrm{mg} / \mathrm{ml}$ MTT reagent (Sigma, St. Louis, MO, USA) and read at $590 \mathrm{~nm}$ (Tecan, Durham, NC, USA).

Transwell assay. MDA-MB-231 and MDA-MB-436 cells were seeded into 12-well plates and transfected with siRNAs against HOXA11-AS in triplicate. After treatment for $48 \mathrm{~h}$, cells were washed, trypsinized and approximate $1 \times 10^{5}$ cells were seeded into the upper chamber in media without fetal bovine serum (FBS). Afterwards, a total of $600 \mu \mathrm{l}$ of complete media (supplied with 10\% FBS) were poured into the lower chamber. For the invasion assay, the membrane was pre-coated with the Matrigel (Corning Inc., Acton, MA, USA) for $6 \mathrm{~h}$ at $37^{\circ} \mathrm{C}$. Chambers were then incubated for $24 \mathrm{~h}$. Afterwards, cells were fixed with ice-cold methanol for $5 \mathrm{~min}$. After two washes with PBS, the membrane was stained with crystal violet $(1 \%)$ for $5 \mathrm{~min}$. Top chambers were cleaned with cotton swab and the lower chamber was photographed under a Nikon microscope (Nikon Corporation) with 5 non-biased image fields.

Wound-healing assay. Wound-healing assays were performed by creating identical wound area for anchorage-dependent cells MDA-MB-231 and MDA-MB-436 by $10 \mu 1$ sterile pipette tips. Briefly, cells were seeded into 6-well plates and co-incubated with same amount of siRNAs for $48 \mathrm{~h}$. Afterwards, cells were washed with PBS and scraped a cross in the center of each well, washed with PBS again and replaced with fresh serum-free medium immediately. The cells were photographed once the scratch was made. After $24 \mathrm{~h}$ growth, cells were also observed and photographed under a Nikon microscope (Nikon Corporation, Tokyo, Japan) at a magnification of $\mathrm{x} 200$ for each group. 
Statistically analysis. All data were presented as mean \pm standard deviation (SD). Experiments were repeated at least three times in triplicate. The Student's t-test was used to compare the difference between groups. Any difference with a value of $\mathrm{P}<0.05$ was considered statistically significant.

\section{Results}

LncRNA HOXA11-AS was overexpressed in human breast cancer in vivo and in vitro. To assess the role of HOXA11-AS in breast cancer, we first examined the relative transcript level of HOXA11-AS in the cancerous tissues from 100 breast cancer patients. Of all the 100 patients, only 9 patients showed lower expression of HOXA11-AS in the cancerous tissues compared with their adjacent non-cancerous counterparts. The average transcript level of HOXA11-AS in tumor tissues was remarkably higher (2.7-fold) than the control tissues (Fig. 1A). We subclassified the expression level of HOXA11-AS in these 100 cases as low (lower than the average level) and high (over the average level), and found that the expression of HOXA11-AS was significantly correlated with tumor size, metastasis and TNM staging, while it was not associated with age at onset, marriage status nor histology typing (Table I). Furthermore, clinical statistics revealed that the expression of HOXA11-AS was associated with Ki67 and HER2, but did not correlate with that of ER and PR (Table II). Afterwards, we also detected the in vitro expression of HOXA11-AS. MCF10A is a normal epithelial cell line and used here as a control. It was observed that all of the four breast cancer cell lines showed higher transcript level of HOXA11-AS, of which MDA-MB-231 and MDA-MB-436 cells showed their highest transcript levels by up to 4.41-fold and 3.98-fold, respectively as compared with control MCF10A cells (Fig. 1B). Furthermore, it was well-known that MDA-MB-231 and MDA-MB-436 cells showed higher abilities of migration and invasion, evidenced by other literatures (19-21) and our data (not shown). Thus, these two cell lines were selected for the following studies. These data suggested that the transcript level of HOXA11-AS was increased in human breast cancer.

Knockdown of HOXA11-AS in breast cancer cells inhibited colony formation and arrested cell cycle in G0/G1 phase. Two specific siRNAs against HOXA11-AS were designed and transfected into MDA-MB-231 and MDA-MB-436 cells. As shown in Fig. 2A, the expression of HOXA11-AS was decreased by approximate $50 \%$ in both cell lines by the two siRNAs. Then, the role of HOXA11-AS in cell proliferation was explored. To this end, colony formation assay was performed in the breast cancer cell lines. It was shown that about 175 colonies were counted in control MDA-MB-231 cells, however, only 80 (siHOXA11-AS-1) and 84 (siHOXA11-AS-2) colonies were observed after siRNAs transfection (Fig. 2B). Similar phenomenon was also noticed in MDA-MB-436 cells. Afterwards, it was shown in the cell cycle analysis that transfection of siHOXA11-AS in MDA-MB-231 cells increased the cell percentage in G0/G1 phase by $10 \%$ and decreased cells in $\mathrm{S}$ phase by $8 \%$, while the cell percentage in $\mathrm{G} 2 / \mathrm{M}$ phase decreased slightly (Fig. 2C). The cells in G0/G1 phase increased by $13 \%$ by siHOXA11-AS-1 and $11 \%$

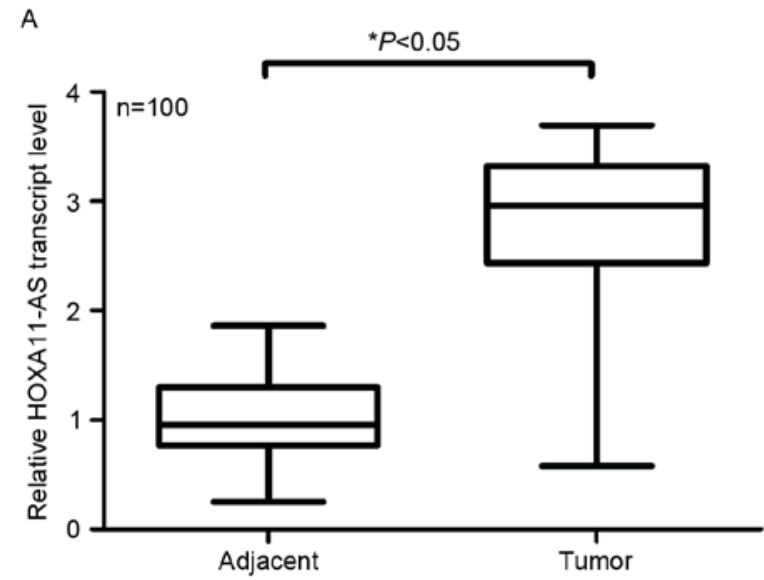

B

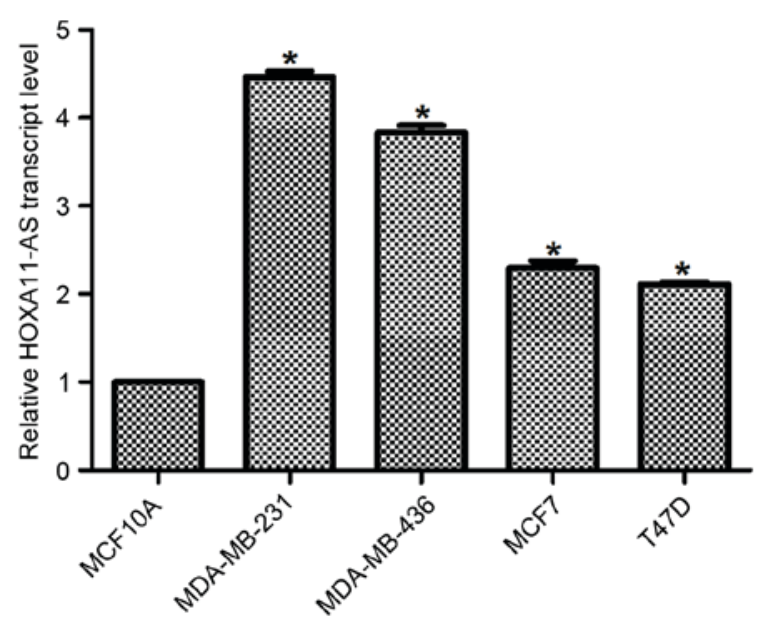

Figure 1. Long non-coding RNA HOXA11-AS was overexpressed in human breast cancer in vivo and in vitro. (A) The relative transcript level of HOAX11-AS was examined in the tumor tissues and their adjacent non-cancerous tissues from 100 clinical patients. ${ }^{*} \mathrm{P}<0.05$, vs. Adjacent. (B) The relative transcript level of HOAX11-AS was examined in four of the breast cancer cell lines MDA-MB-231, MDA-MB-436, MCF7 and T47D as well as a normal epithelial cell line MCF10A. ${ }^{*} \mathrm{P}<0.05$, vs. MCF10A. HOXA11-AS, homeobox A11-AS.

by siHOXA11-AS-2, respectively in MDA-MB-436 cells. Accordingly, the cell percentage in $\mathrm{S}$ phase was decreased significantly and that in G2/M phase also decreased slightly (Fig. 2D). These data suggested that depletion of HOXA11-AS in human breast cancer cells inhibited colony formation and suppressed cell proliferation.

Knockdown of HOXA11-AS suppressed cell proliferation in human breast cancer cells. Next, cell proliferative rate was explored when MDA-MB-231 and MDA-MB-436 cells were transfected with siHOXA11-AS. There were no significant differences among the four groups in the former three days for both cell lines. However, on the fourth day, the cell proliferative rate was retarded by $36 \%$ with either siRNA against HOXA11-AS, and the effects went stronger on the fifth day in MDA-MB-231 cells (Fig. 3A). Likewise, transfection of siHOXA11-AS decreased the cell proliferative rate by $20 \%$ and approximate $28 \%$, on the fourth day and fifth day, 
Table I. Association of homeobox A11-AS with clinical variables among 100 female breast cancer patients.

\begin{tabular}{|c|c|c|c|c|}
\hline \multirow[b]{2}{*}{ Variables } & \multirow[b]{2}{*}{ Numbers } & \multicolumn{2}{|c|}{ Expression of homeobox A11-AS } & \multirow[b]{2}{*}{ P-value } \\
\hline & & Low $(n=54)$ & $\operatorname{High}(\mathrm{n}=46)$ & \\
\hline Age (years) & & & & 0.630 \\
\hline$<40$ & 29 & 18 & 11 & \\
\hline $40-55$ & 33 & 17 & 16 & \\
\hline$>55$ & 38 & 19 & 19 & \\
\hline Marriage status & & & & 0.124 \\
\hline Single & 16 & 7 & 9 & \\
\hline Married & 51 & 25 & 26 & \\
\hline Widowed & 24 & 18 & 6 & \\
\hline Divorced/separated & 9 & 4 & 5 & \\
\hline Tumor size $(\mathrm{T})$ & & & & $<0.001$ \\
\hline $\mathrm{T} 1(\leq 2 \mathrm{~cm})$ & 14 & 3 & 11 & \\
\hline $\mathrm{T} 2(>2 \mathrm{~cm}$ but $<5 \mathrm{~cm})$ & 23 & 4 & 19 & \\
\hline $\mathrm{T} 3(\geq 5 \mathrm{~cm})$ & 21 & 10 & 11 & \\
\hline $\mathrm{T} 4$ (any size with distant metastasis) & 42 & 37 & 5 & \\
\hline Lymph node metastasis $(\mathrm{N})$ & & & & 0.422 \\
\hline No & 51 & 30 & 21 & \\
\hline N1 or above & 49 & 24 & 25 & \\
\hline Distant metastasis (M) & & & & $<0.001$ \\
\hline M0 & 58 & 17 & 41 & \\
\hline M1 & 42 & 37 & 5 & \\
\hline TNM stage & & & & $<0.001$ \\
\hline I/II & 42 & 11 & 31 & \\
\hline III/IV & 58 & 43 & 15 & \\
\hline Histology & & & & 0.351 \\
\hline Ductal & 11 & 4 & 7 & \\
\hline Lobular & 52 & 31 & 21 & \\
\hline Mixed & 33 & 18 & 15 & \\
\hline Other & 4 & 1 & 3 & \\
\hline
\end{tabular}

Table II. Association of lncRNA homeobox A11-AS with pathological variables among 100 breast cancer patients.

\begin{tabular}{|c|c|c|c|c|}
\hline \multirow[b]{2}{*}{ Variables } & \multirow[b]{2}{*}{ Numbers } & \multicolumn{2}{|c|}{ Expression of homeobox A11-AS } & \multirow[b]{2}{*}{ P-value } \\
\hline & & Low $(\mathrm{n}=54)$ & High $(n=46)$ & \\
\hline Ki67 & & & & 0.001 \\
\hline$<14 \%$ & 44 & 16 & 28 & \\
\hline$\geq 14 \%$ & 56 & 39 & 17 & \\
\hline ER & & & & 0.305 \\
\hline Negative & 61 & 23 & 38 & \\
\hline Positive & 39 & 19 & 20 & \\
\hline PR & & & & 0.414 \\
\hline Negative & 59 & 30 & 29 & \\
\hline Positive & 41 & 25 & 16 & \\
\hline HER-2 & & & & 0.003 \\
\hline Negative & 54 & 21 & 33 & \\
\hline Positive & 46 & 32 & 14 & \\
\hline
\end{tabular}

ER, estrogen receptor; PR, progesterone receptor; HER-2, human epidermal growth factor receptor-2. 

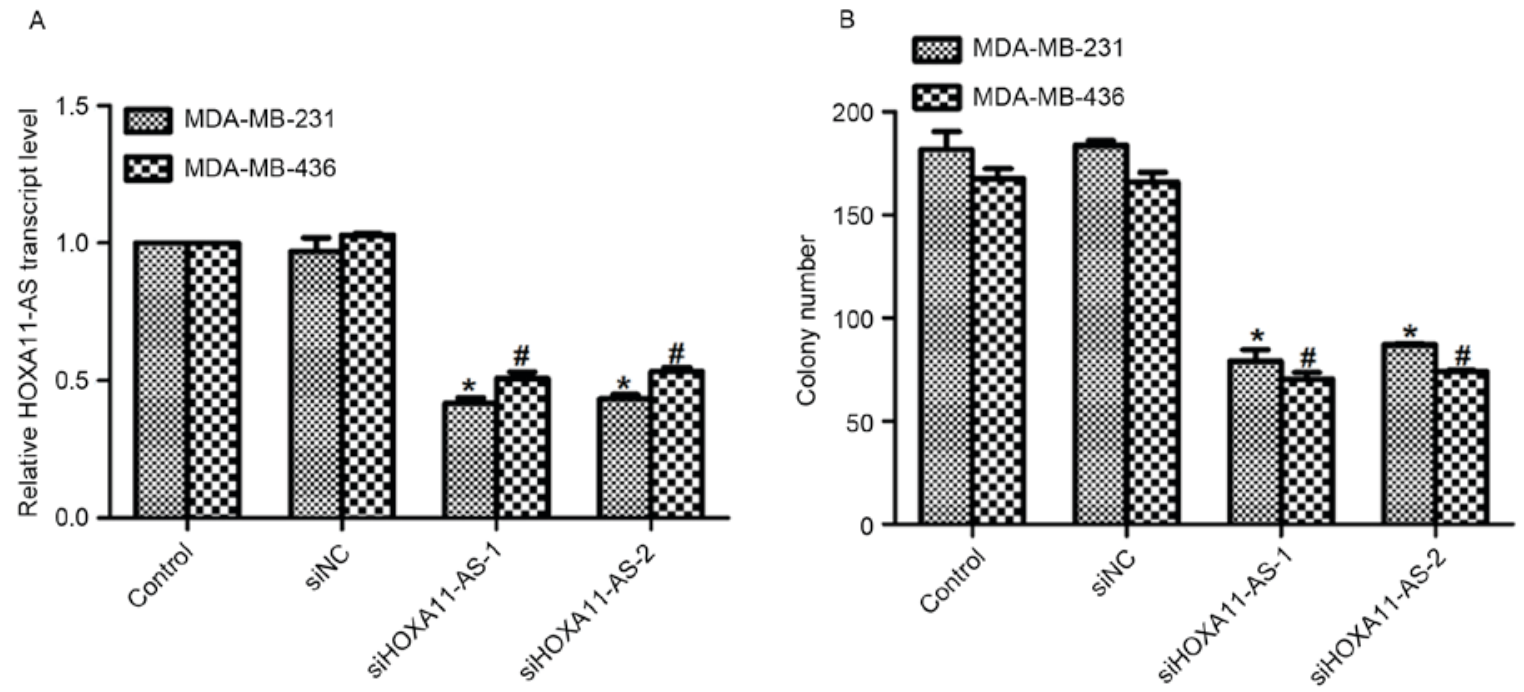

C
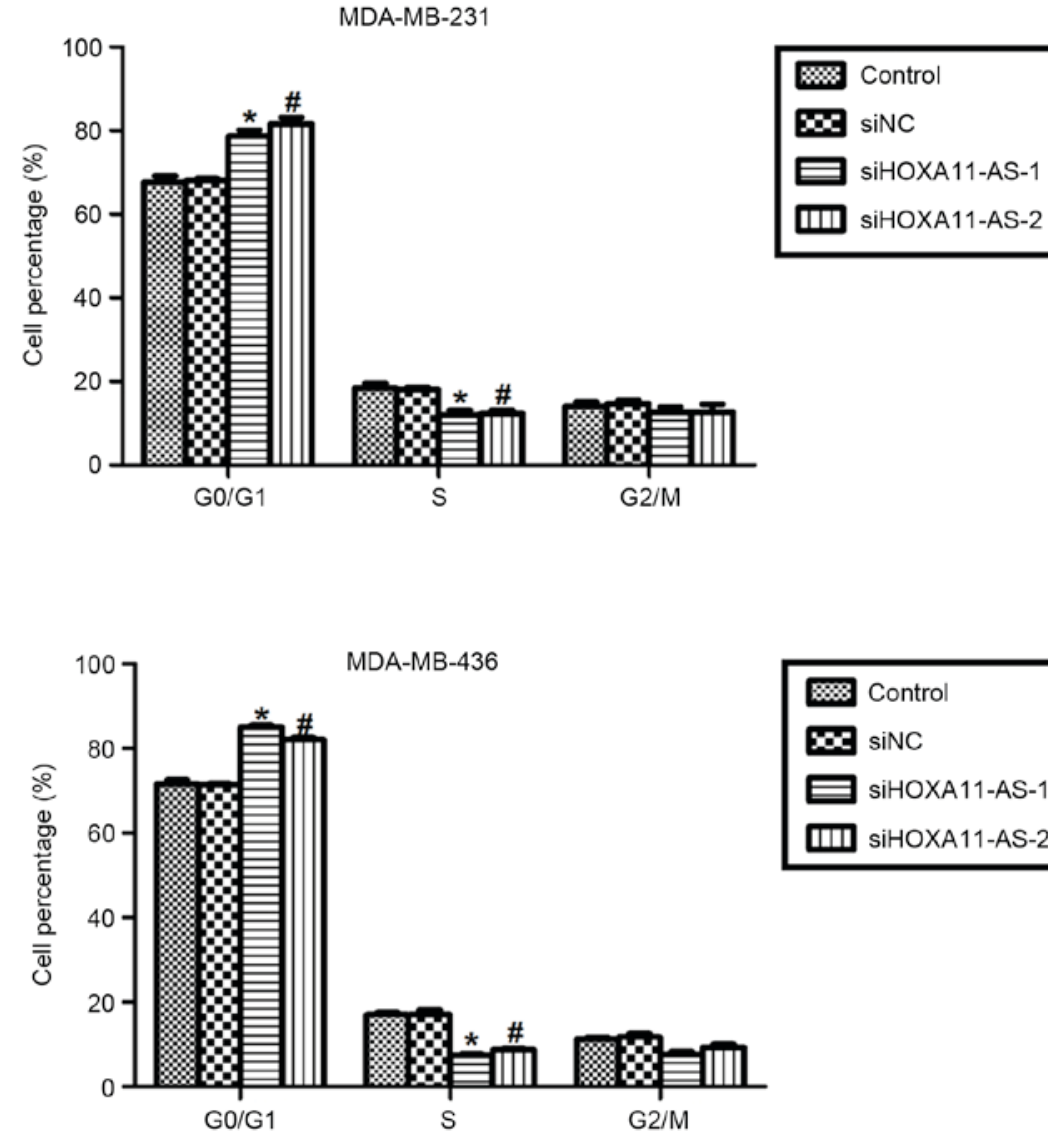

Figure 2. Knockdown of HOXA11-AS in breast cancer cells inhibited colony formation and arrested cell cycle in G0/G1 phase. (A) Two specific siRNAs against HOXA11-AS were transfected into MDA-MB-231 and MDA-MB-436 cells and the relative expression of HOXA11-AS was explored. (B) Colony formation assays were performed to explore the effects of depletion of HOXA11-AS on colony formation in MDA-MB-231 and MDA-MB-436 cells ${ }^{*} \mathrm{P}<0.05$ vs. Control in MDA-MB-231 cells. ${ }^{~} \mathrm{P}<0.05$ vs. Control in MDA-MB-436 cells. (C) Cell cycle analysis was performed in MDA-MB-231 cells when cells were transfected with siRNAs against HOXA11-AS and the cell percentage in each phase was calculated. (D) Cell cycle analysis was performed in MDA-MB-436 cells when cells were transfected with siRNAs against HOXA11-AS and the cell percentage in each phase was calculated. "P<0.05 vs. Control in siHOXA11-AS-1 treated cells. ${ }^{\text {PP }}<0.05$ vs. Control in siHOXA11-AS-2 treated cells. siNC, negative control siRNA; siHOXA11-AS, specific siRNA against lncRNA homeoboxA11-AS.

respectively (Fig. 3B). These results showed that knockdown of HOXA11-AS inhibited cell proliferation in human breast cancer.
Knockdown of HOXA11-AS inhibited cell migration and invasion in breast cancer cells. Cell proliferation and metastasis are two main manifestations of malignancies. Thus, we 
A

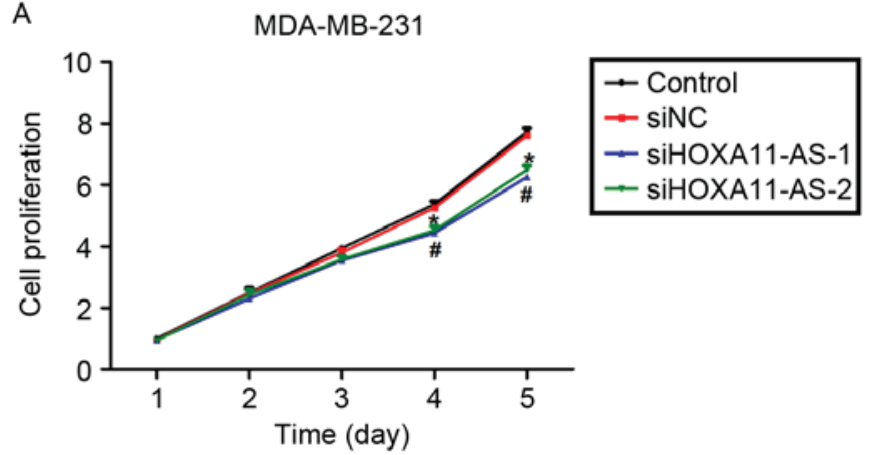

B

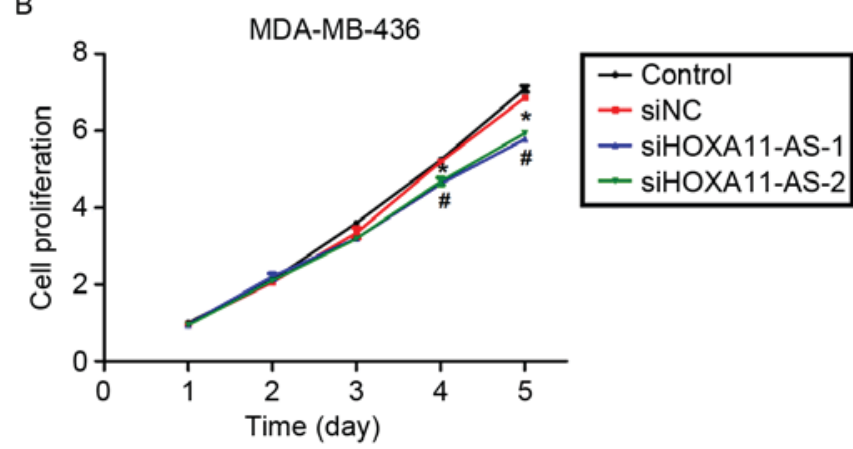

Figure 3. Knockdown of HOXA11-AS suppressed cell proliferation in human breast cancer cells. (A) Cell proliferation assay was performed in MDA-MB-231 cells when cells were transfected with siRNAs against HOXA11-AS in a consecutive five days. (B) Cell proliferation assay was performed in MDA-MB-436 cells when cells were transfected with siRNAs against HOXA11-AS in a consecutive five days. ${ }^{*} \mathrm{P}<0.05$ vs. Control in siHOXA11-AS-1 treated cells. ${ }^{\#} \mathrm{P}<0.05$ vs. Control in siHOXA11-AS-2 treated cells. siNC, negative control siRNA; siHOXA11-AS, specific siRNA against lncRNA HOXA11-AS.

A

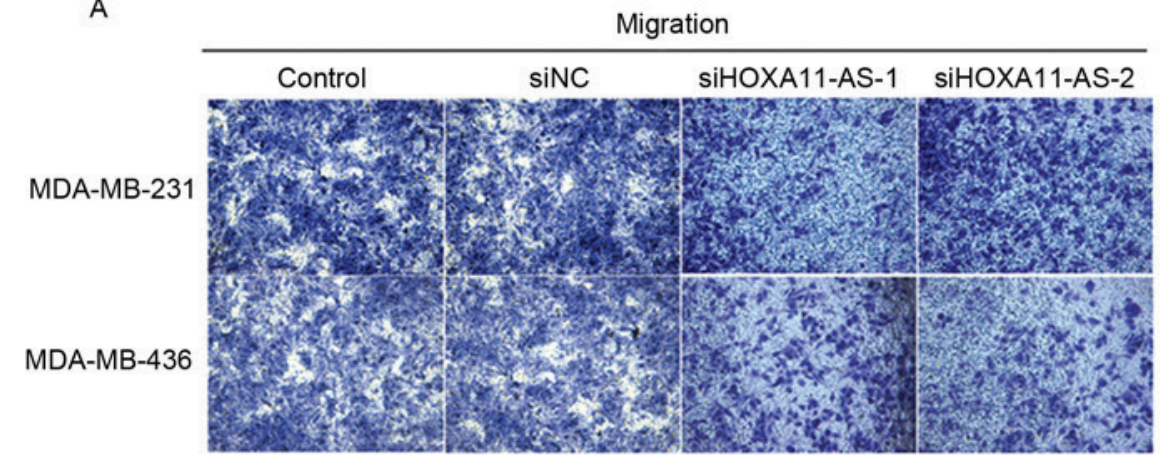

C

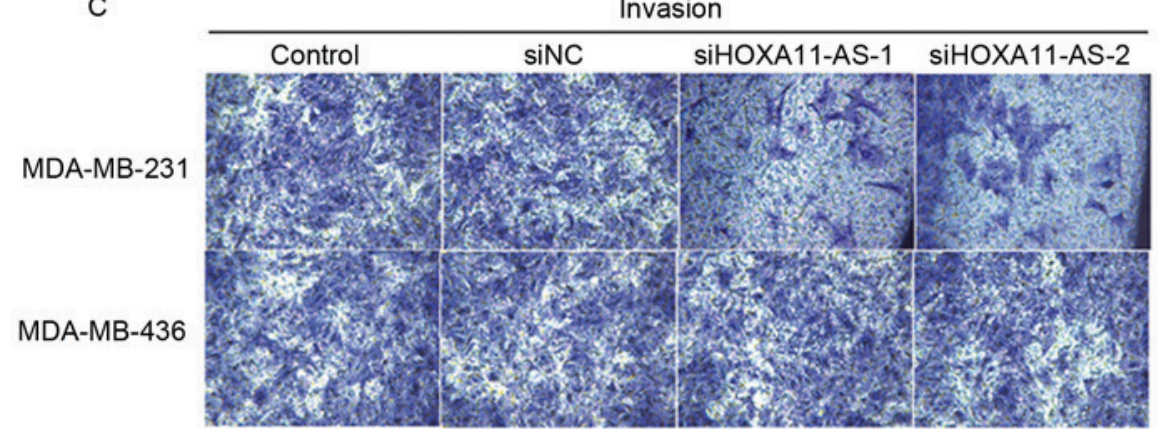

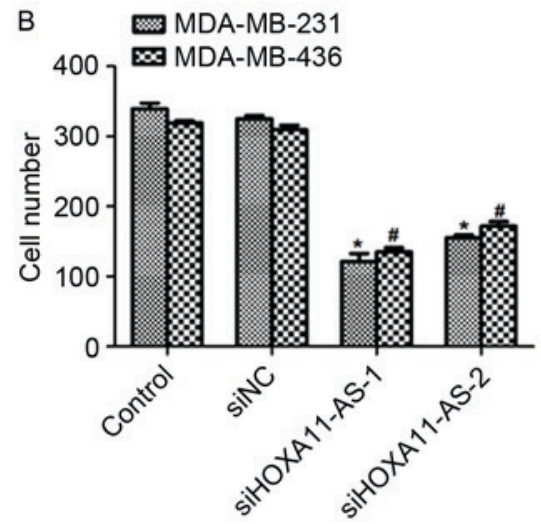

D

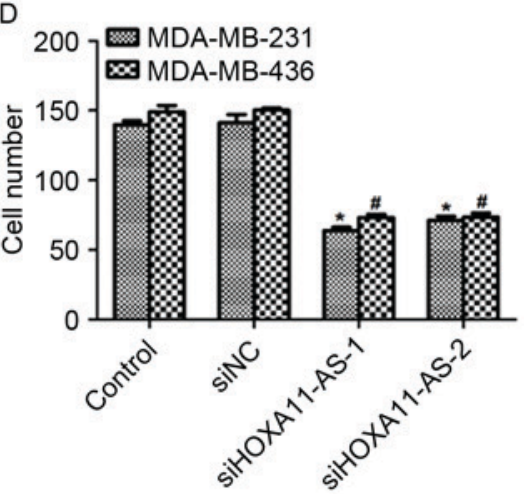

Figure 4. Knockdown of HOXA11-AS inhibited cell migration and invasion in breast cancer cells. (A) Representative images of cell migration assays in MDA-MB-231 and MDA-MB-436 cells when cells were treated with siRNAs against HOXA11-AS. (B) Quantification of cell migration assays in MDA-MB-231 and MDA-MB-436 cells by counting cells migrated through the membranes. ${ }^{*} \mathrm{P}<0.05$ vs. Control in MDA-MB-231 cells. ${ }^{~} \mathrm{P}<0.05$ vs. Control in MDA-MB-436 cells. (C) Representative images of cell invasion assays in MDA-MB-231 and MDA-MB-436 cells when cells were treated with siRNAs against HOXA11-AS. (D) Quantification of cell invasion assays in MDA-MB-231 and MDA-MB-436 cells by counting cells on the lower surface of the chamber. "P<0.05 vs. Control in MDA-MB-231 cells. "P<0.05, vs. Control in MDA-MB-436 cells. siNC, negative control siRNA; siHOXA11-AS, specific siRNA against lncRNA homeoboxA11-AS.

further explored the effects of HOXA11-AS on cell metastasis by transwell assays and wound-healing assays. In cell migration assays, more than 300 MDA-MB-231 cells were observed to migrate through the membrane in the control group; however, only 125 and 136 cells were able to migrate onto the lower surface of the chamber in siHOXA11-AS-1 and siHOXA11-AS-2 treated cells, respectively, while siNC caused no effects on cell migration (Fig. 4A and B). Similar observation was also demonstrated in MDA-MB-436 cells when cells were treated with either specific siRNAs. Next, cell invasion assay showed that siHOXA11-AS-1 caused decreases of approximate 80 MDA-MB-231 cells and 76 MDA-MB-436 cells, respectively, which invaded onto the upper surface of the chamber. Similarly, siHOXA11-AS-2 also decreased cell invasive abilities by up to $60 \%$ in MDA-MB-231 and $53 \%$ in MDA-MB-436 cells (Fig. 4C and D). 
A

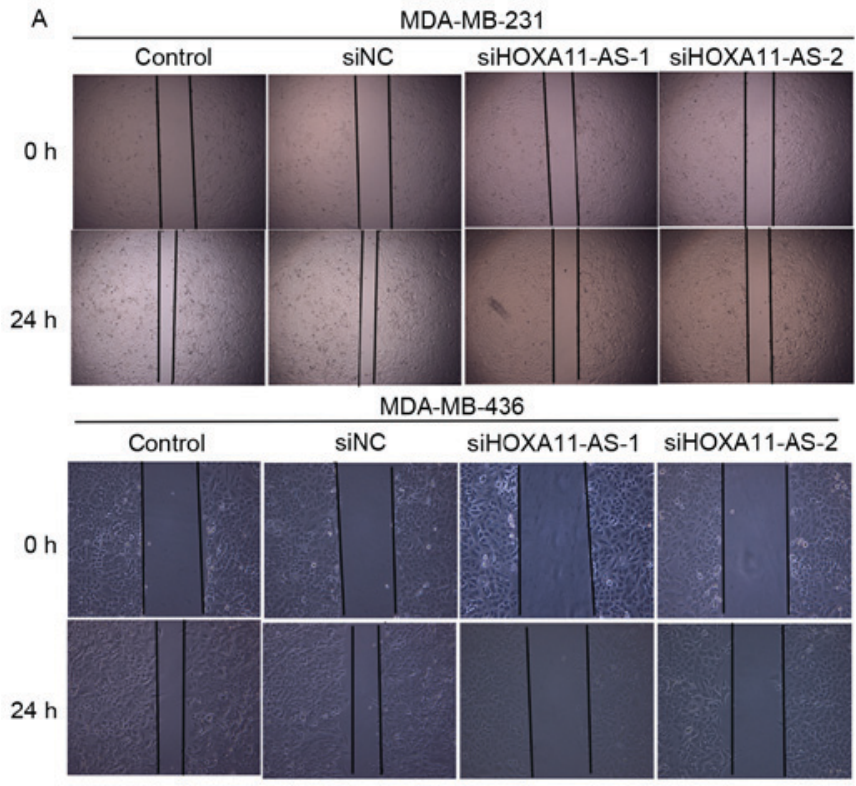

B

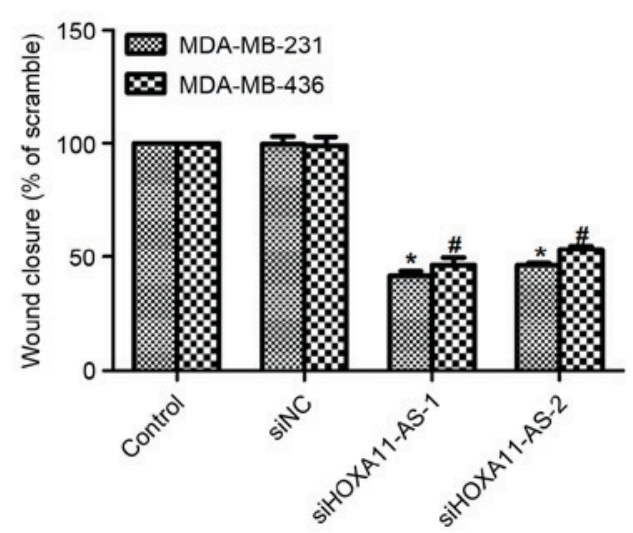

Figure 5. Knockdown of HOXA11-AS inhibited cell metastasis in breast cancer cell lines. (A) Representative images of wound-healing assays in MDA-MB-231 and MDA-MB-436 cells when cells were treated with siRNAs against HOXA11-AS. (B) Quantification of wound-healing assays in MDA-MB-231 and MDA-MB-436 cells by calculating the area of the wound closure in each treating group. ${ }^{*} \mathrm{P}<0.05$ vs. Control in MDA-MB-231 cells. ${ }^{\#} \mathrm{P}<0.05$ vs. Control in MDA-MB-436 cells. siNC, negative control siRNA; siHOXA11-AS, specific siRNA against lncRNA homeoboxA11-AS.

In the wound-healing assays the abilities of both cell lines to close the wound scraped in the middle of the cultural plate were notably inhibited (Fig. 5A). Quantification of wound-healing assay showed that the wound closure was suppressed by more than $50 \%$ by either specific siRNA in MDA-MB-231 cells and MDA-MB-436 cells (Fig. 5B). All of these data suggested that knockdown of HOXA11-AS in breast cancer cells inhibited cell metastasis in vitro.

\section{Discussion}

Breast cancer is one of the most common cancers in females. According to the latest investigation by NCI (National Cancer Institute), there are 246,660 new cases of breast cancer in 2016 which account for $14.6 \%$ of all new cancer cases worldwide. Among these cases, 40,450 cases (approximate 6.8\%) are estimated to die. Moreover, it has been reported that in 2013 there are 3,053,450 females living with breast cancer which significantly affect the life quality of these patients $(4,22)$. Therefore, it is urgently needed to discover novel therapeutic strategies against breast cancer.

The LncRNA HOXA11-AS was initially characterized using the RNA-seq analysis (23). The present study found that LncRNA HOXA11-AS was upregulated in breast cancer both in vivo and in vitro. Afterwards, we explored the detailed roles of HOXA11-AS in cell proliferation and cell metastasis in breast cancer cell lines. MDA-MB-231 and MDA-MB-436 cells were selected as the cell models in the current study due to their highest expression profiles of HOXA11-AS (Fig. 1B). Since the expression of HOXA11-AS did not correlate with ER nor PR (Table II), we did not use other breast cancer cell lines that are ER-positive. Moreover, two specific siRNAs against HOXA11-AS were designed and transfected into the cells to deplete the expression of HOXA11-AS. However, the limitation of this study is that the overexpression plasmid was not included. In the future study, we will clone the long sequence of HOXA11-AS into the blank vector pcDNA 3.1 and transfected this expressing plasmid into those cells with a lower HOXA11-AS expression.

Cell proliferation and cell metastasis are two main manifestation of malignancies. Thus, we determined the roles of HOXA11-AS by colony formation assay, cell cycle assay, cell proliferation assay, transwell assay and wound-healing assay, and found that knockdown of HOXA11-AS in MDA-MB-231 and MDA-MB-436 cells significantly inhibited cell proliferation and metastasis, which was consistent with other researchers' findings $(16,24,25)$. In addition, Wang et al (18) showed that HOXA11-AS was closely associated with glioma grading and positively related with poor prognosis. In our study, clinical statistics revealed that the expression of HOXA11-AS significantly correlated with clinical variables such as tumor size, distant metastasis and TNM staging (Table I). The index $\mathrm{Ki} 67$ which is an indicator for cell growth also correlates with the expression of HOXA11-AS (Table II). Our clinical data validated the in vitro functional observations of HOXA11-AS in breast cancer cells and was also similar with the findings by Wang et al (18). Together with the previous reports, it could be conceived that HOXA11-AS might serve as a critical clinical biomarker in human tumorigenesis. However, the limitation of this study was that the effects of HOXA11-AS on cell proliferation and metastasis were only explored in MDA-MB-231 and MDA-MB-436 cells, which showed higher expression of HOXA11-AS. We are now trying to include other cell lines to uncover the mechanisms of how HOXA11-AS functions in breast cancer. And this exploration would be in full discussion in our subsequent studies.

It would be interesting to explore the detailed mechanisms of how HOXA11-AS exerts its biological activity in breast cancer. It is currently scanty on this mechanism studies. A recent high-throughput sequencing analysis showed that HOXA11-AS silencing highlighted alterations in cell proliferation and cell-cell adhesion pathways. Mechanistically, EZH2 along with the histone demethylase LSD1 or DNMT1 were recruited by HOXA11-AS, which functioned as a scaffold. HOXA11-AS also functioned as a molecular sponge for miR-1297, antagonizing its ability to repress EZH2 protein translation (17). Furthermore, a comprehensive analysis of HOXA11-AS gene interaction regulatory network revealed that 
HOXA11-AS regulates the expression of various pathways and genes, especially DOCK8 and TGF-beta pathway (26). These pioneer findings indicated that cell proliferation and cell-cell adhesion pathways were critically involved in HOXA11-AS functions, though its detailed regulatory molecular mechanism might differ across different cancer types. This also prompts us to further study on the mechanisms of how HOX11-AS works in breast cancer and compare the pathway involvement of HOXA11-AS across different cancer types.

In total, our data suggested that the transcript level of HOXA11-AS was highly upregulated in breast cancer both in vivo and in vitro. Knockdown of HOXA11-AS in MDA-MB-231 and MDA-MB-436 cells inhibited colony formation and cell proliferative rate, and caused cell cycle arrest in G0/G1 phase. In addition, depletion of HOXA11-AS with specific siRNAs suppressed cell migration and invasion in breast cancer cells. Our findings indicated the oncogenic potential of HOXA11-AS in human breast cancer and provided new evidence for the diagnosis and treatment of breast cancer in clinic.

\section{References}

1. Tesarova P: Specific aspects of breast cancer therapy of elderly women. Biomed Res Int 2016: 1381695, 2016.

2. Hannoun-Levi JM, Courdi A, Marsiglia H, Namer M and Gerard JP: Breast cancer in elderly women: Is partial breast irradiation a good alternative? Breast Cancer Res Treat 81: 243-251, 2003.

3. Harding C, Pompei F, Burmistrov D, Welch HG, Abebe R and Wilson R: Breast cancer screening, incidence and mortality across US counties. JAMA Intern Med 175: 1483-1489, 2015.

4. Belot A, Grosclaude P, Bossard N, Jougla E, Benhamou E, Delafosse P, Guizard AV, Molinié F, Danzon A, Bara S, et al: Cancer incidence and mortality in France over the period 1980-2005. Rev Epidemiol Sante Publique 56: 159-175, 2008.

5. Ikeda $A$, Kawachi I, Iso $H$, Iwasaki $M$, Inoue $M$ and Tsugane S: Social support and cancer incidence and mortality: The JPHC study cohort II. Cancer Causes Control 24: 847-860, 2013.

6. Miller BA, Chu KC, Hankey BF and Ries LA: Cancer incidence and mortality patterns among specific Asian and Pacific Islander populations in the U.S. Cancer Causes Control 19: 227-256, 2008.

7. Sas-Chen A, Aure MR, Leibovich L, Carvalho S, Enuka Y, Körner C, Polycarpou-Schwarz M, Lavi S, Nevo N, Kuznetsov Y, et al: LIMT is a novel metastasis inhibiting lncRNA suppressed by EGF and downregulated in aggressive breast cancer. EMBO Mol Med 8: 1052-1064, 2016.

8. Lee JJ, Kim M and Kim HP: Epigenetic regulation of long noncoding RNA UCA1 by SATB1 in breast cancer. BMB Rep 49: $578-583,2016$

9. Van Grembergen O, Bizet M, de Bony EJ, Calonne E, Putmans P, Brohée S, Olsen C, Guo M, Bontempi G, Sotiriou C, et al: Portraying breast cancers with long noncoding RNAs. Sci Adv 2: e1600220, 2016.

10. Huang YS, Chang CC, Lee SS, Jou YS and Shih HM: Xist reduction in breast cancer upregulates AKT phosphorylation via HDAC3-mediated repression of PHLPP1 expression. Oncotarget 7: 43256-43266, 2016.
11. Zhang Y, He Q, Hu Z, Feng Y, Fan L, Tang Z, Yuan J, Shan W, $\mathrm{Li}$ C, Hu X, et al: Long noncoding RNA LINP1 regulates repair of DNA double-strand breaks in triple-negative breast cancer. Nat Struct Mol Biol 23: 522-530, 2016

12. Mainguy G, Koster J, Woltering J, Jansen H and Durston A: Extensive polycistronism and antisense transcription in the mammalian Hox clusters. PLoS One 2: e356, 2007.

13. Krumlauf R: Hox genes in vertebrate development. Cell 78: 191-201, 1994.

14. Fiegl H, Windbichler G, Mueller-Holzner E, Goebel G, Lechner M, Jacobs IJ and Widschwendter M: HOXA11 DNA methylation-a novel prognostic biomarker in ovarian cancer. Int J Cancer 123: 725-729, 2008.

15. Chau YM, Pando S and Taylor HS: HOXA11 silencing and endogenous HOXA11 antisense ribonucleic acid in the uterine endometrium. J Clin Endocrinol Metab 87: 2674-2680, 2002.

16. Kim HJ, Eoh KJ, Kim LK, Nam EJ1 Yoon SO, Kim KH, Lee JK, Kim SW and Kim YT: The long noncoding RNA HOXA11 antisense induces tumor progression and stemness maintenance in cervical cancer. Oncotarget 7: 83001-83016, 2016.

17. Sun M, Nie F, Wang Y, Zhang Z, Hou J, He D, Xie M, Xu L, De W, Wang Z and Wang J: LncRNA HOXA11-AS promotes proliferation and invasion of gastric cancer by scaffolding the chromatin modification factors PRC2, LSD1, and DNMT1. Cancer Res 76: 6299-6310, 2016

18. Wang Q, Zhang J, Liu Y, Zhang W, Zhou J, Duan R, Pu P, Kang C and Han L: A novel cell cycle-associated lncRNA, HOXA11-AS, is transcribed from the 5-prime end of the HOXA transcript and is a biomarker of progression in glioma. Cancer Lett 373: 251-259, 2016.

19. Li T, Liu Y, Xiao H and Xu G: Long non-coding RNA TUG1 promotes cell proliferation and metastasis in human breast cancer. Breast Cancer: Nov 15, 2016 (Epub ahead of print).

20. Jia Z, Liu Y, Gao Q, Han Y, Zhang G, Xu S, Cheng K and Zou W: miR-490-3p inhibits the growth and invasiveness in triple-negative breast cancer by repressing the expression of TNKS2. Gene 593: 41-47, 2016.

21. Zhou T, Zhang B, Wei P, Du Y, Zhou H, Yu M, Yan L, Zhang W, Nie G, Chen C, et al: Energy metabolism analysis reveals the mechanism of inhibition of breast cancer cell metastasis by PEG-modified graphene oxide nanosheets. Biomaterials 35: 9833-9843, 2014.

22. Banas T, Juszczyk G, Pitynski K, Nieweglowska D, Ludwin A and Czerw A: Incidence and mortality rates in breast, corpus uteri, and ovarian cancers in Poland (1980-2013): An analysis of population-based data in relation to socioeconomic changes. Onco Targets Ther 9: 5521-5530, 2016.

23. Chen J, Fu Z, Ji C, Gu P, Xu P, Yu N, Kan Y, Wu X, Shen R and Shen Y: Systematic gene microarray analysis of the lncRNA expression profiles in human uterine cervix carcinoma. Biomed Pharmacother 72: 83-90, 2015.

24. Connell KA, Guess MK, Chen H, Andikyan V, Bercik R and Taylor HS: HOXA11 is critical for development and maintenance of uterosacral ligaments and deficient in pelvic prolapse. J Clin Invest 118: 1050-1055, 2008.

25. Se YB, Kim SH, Kim JY, Kim JE, Dho YS, Kim JW, Kim YH, Woo HG, Kim SH, Kang SH, et al: Underexpression of HOXA11 is associated with treatment resistance and poor prognosis in Glioblastoma. Cancer Res Treat 49: 387-398, 2017.

26. Zhang Y, He RQ, Dang YW, Zhang XL, Wang X, Huang SN, Huang WT, Jiang MT, Gan XN, Xie Y, et al: Comprehensive analysis of the long noncoding RNA HOXA11-AS gene interaction regulatory network in NSCLC cells. Cancer Cell Int 16: 89, 2016. 